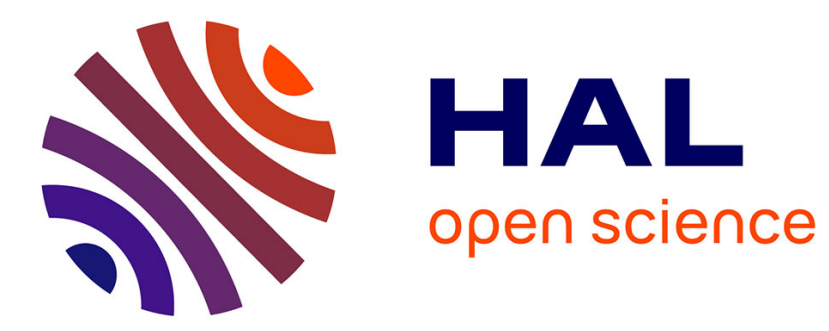

\title{
Optical reflectivity of a diffuse interface
}

\author{
J. Meunier, D. Langevin
}

\section{- To cite this version:}

J. Meunier, D. Langevin. Optical reflectivity of a diffuse interface. Journal de Physique Lettres, 1982, 43 (6), pp.185-191. 10.1051/jphyslet:01982004306018500 . jpa-00232029

\section{HAL Id: jpa-00232029 https://hal.science/jpa-00232029}

Submitted on 1 Jan 1982

HAL is a multi-disciplinary open access archive for the deposit and dissemination of scientific research documents, whether they are published or not. The documents may come from teaching and research institutions in France or abroad, or from public or private research centers.
L'archive ouverte pluridisciplinaire HAL, est destinée au dépôt et à la diffusion de documents scientifiques de niveau recherche, publiés ou non, émanant des établissements d'enseignement et de recherche français ou étrangers, des laboratoires publics ou privés. 
Classification

Physics Abstracts

$07.60 \mathrm{H}-64.70 \mathrm{~J}-68.10-82.70$

\title{
Optical reflectivity of a diffuse interface
}

\author{
J. Meunier and D. Langevin \\ Laboratoire de Physique de l'Ecole Normale Supérieure, \\ 24, rue Lhomond, 75231 Paris Cedex 05, France
}

(Reçu le 24 novembre 1981, accepté le 28 janvier 1982)

\begin{abstract}
Résumé. - Nous avons calculé la réflectivité d'un interface entre deux fluides en tenant compte des fluctuations thermiques, négligées par de précédents auteurs. Nous présentons une nouvelle interprétation de leurs résultats, ainsi que de nouvelles mesures de réflectivité sur des mélanges près de points critiques de démixtion, présentant des phases microémulsions. La contribution des fluctuations thermiques à l'épaisseur d'interface peut être dominante dans certains cas.
\end{abstract}

\begin{abstract}
We have computed the reflectivity of an interface between two fluids taking into account the thermal fluctuations at the interface, neglected by former authors. We present a new interpretation of their data together with new reflectivity measurements that we have made in multiphase microemulsion systems close to critical consolute points. The contribution of the thermally excited surface waves to interfacial thickness can be dominant in some cases.
\end{abstract}

1. Introduction. - Far from a critical point the interface between two fluid phases is very sharp. But as a critical point is approached, the interface thickens and becomes diffuse, until, at the critical point, it disappears completely, the two phases becoming identical. The classical density profile of the Van der Waals [1] and Cahn-Hilliard [2] theories is described by :

$$
\rho(z)=\frac{1}{2}\left[\rho_{1}+\rho_{2}+\left(\rho_{1}-\rho_{2}\right) \tanh (2 z / L)\right]
$$

where $\rho(z)$ is the density at height $z, \rho_{1}$ and $\rho_{2}$ the densities of the lower and upper phase respectively. Fisk and Widom [3] have given an alternative formula for $\rho(z)$ :

$$
\rho(z)=\frac{1}{2}\left\{\left(\rho_{1}+\rho_{2}\right)+\left(\rho_{1}-\rho_{2}\right) \frac{\sqrt{2} \tanh \sqrt{6} x / L}{\left[3-\tanh ^{2}(\sqrt{6} x / L)\right]^{1 / 2}}\right\} .
$$

According to scaling arguments $L$ should vary with temperature as :

$$
L=L_{0} \varepsilon^{-v^{\prime}} \quad \varepsilon=\left(T_{\mathrm{c}}-T\right) / T_{\mathrm{c}}
$$

$v^{\prime}$ being the critical exponent of the correlation lengths in the bulk phases below $T_{\mathrm{c}}$ :

$$
\xi^{\prime}=\xi_{0}^{\prime} \varepsilon^{-v^{\prime}} \text { and } L_{0} \sim 2 \sqrt{6} \xi_{0}^{\prime} \quad[3]
$$


Buff, Lovett and Stillinger [4] proposed that the apparent thickness of the interface is due to thermal surface fluctuations : the interface is sharp, but rough. They described the averaged interfacial composition with an error function $\left({ }^{1}\right)$ :

$$
\rho(z)=\frac{1}{2}\left\{\left(\rho_{1}+\rho_{2}\right)+\frac{1}{\sqrt{\pi}}\left(\rho_{1}-\rho_{2}\right) \int_{0}^{z / L \sqrt{2}} \mathrm{e}^{-t^{2}} \mathrm{~d} t\right\}
$$

where $L$ is the mean square displacement of the interface : $L=\left\langle\zeta^{2}\right\rangle^{1 / 2}$,

$$
\left\langle\zeta^{2}\right\rangle=\int_{0}^{q_{\max }} \frac{k_{\mathrm{B}} T}{\gamma q^{2}+\Delta \rho g} \frac{q \mathrm{~d} q}{2 \pi}=\frac{k_{\mathrm{B}} T}{4 \pi \gamma} \ln \left(q_{\max }^{2} l_{\mathrm{c}}^{2}+1\right)
$$

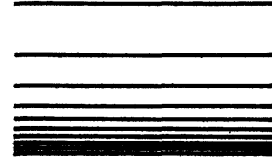

a

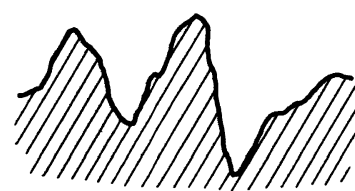

b

Fig. 1. - Interfacial structure in two extreme cases : a) flat and diffuse profile; $b$ ) sharp and rough profile.

with $\Delta \rho=\rho_{1}-\rho_{2} ; \gamma$ is the interfacial tension; $l_{\mathrm{c}}=[\gamma / \Delta \rho g]^{1 / 2}$ is the capillary length; $q_{\max }$ is the largest wave vector of the fluctuations $q_{\max } \sim \pi / L$.

Several years ago, W. W. Webb and collaborators reported on measurements of the optical reflectivity of the interface between two phases in equilibrium just below the critical temperature for both a pure fluid [5] and a binary mixture [6, 7]. By comparison with the Fresnel reflectivity calculated for a sharp boundary they deduced effective thicknesses up to $1000 \AA$ close to the critical temperature. Although the results were not very sensitive to the shape of the density profile, a better agreement was obtained with the error function profile predicted by the BLS theory.

However, the experimental values of $L$ were found to be larger than the mean square amplitude of thermal fluctuations given by equation (4). Conversely the variation of $L$ with temperature was in good agreement with the scaling predictions of Fisk and Widom; the scale factors $\xi_{0}^{\prime}$ and $L_{0}$ were of the same order. Moreover the authors of references [5] to [7] claimed that the thermal fluctuations scatter light at very small angles around the specular reflection and that the scattered light was included in the specular reflectivity measurements. Therefore they did not expect a decrease of the measured reflectivity by the thermal fluctuations (BLS model).

In the following we will show that this approximation is usually very crude. In particular it does not take into account the contribution of the thermal fluctuations of wave vectors $q \lesssim 2 \pi / \lambda$, $\lambda$ being the light wavelength, that do not scatter light, but decrease the reflectivity. We present in this letter a complete calculation of the reflectivity of an interface simultaneously rough and diffuse and a reinterpretation of the data of references [5] and [7]. We also present reflectivity measurements made in multiphase microemulsion systems close to critical consolute points.

(1) Equation (3) describes a diffuse but flat interface. It must be kept in mind that the real interface is sharp and rough (Fig. 1).

$\left({ }^{2}\right)$ The integration region should in fact extend from $q_{\min }$ to $q_{\max }$, where $q_{\min }=\pi / D$, with $D$ the dimension of the system. But in the experiments the condition $q_{\min } l_{\mathrm{c}} \ll 1$ is always satisfied, so that $q_{\min }$ can be replaced by 0 in equation (4). 
2. Reflectivity of a thick intertace. - Thermal motion induces collective surface fluctuations that behave like capillary waves. The mean square amplitude of the vertical displacement of the interface is given by equation (4). Let us introduce the average wave vector of light in the interfacial region : $k=\left(n_{1}+n_{2}\right) \pi / \lambda, n_{1}$ and $n_{2}$ being the refractive indices of the two phases. Among the surface fluctuations those of wave vector smaller than $k$ scatter light whereas those for $q>k$ do not. Let us suppose first that the interface is rough but sharp like in the BLS model. The reflectivity loss due to surface scattering can be calculated by generalizing a result of Beckmann and Spizzichino for perfectly reflecting rough surfaces [8]. One obtains [9] $\left({ }^{3}\right)$ :

$$
R_{1}=R_{\mathrm{F}} \mathrm{e}^{-4 k^{2}\left\langle\zeta_{1}^{2}\right\rangle}
$$

where $R_{\mathrm{F}}$ is the Fresnel reflectivity corresponding to flat and sharp interfaces and $\left\langle\zeta_{1}^{2}\right\rangle$ represent the contribution of the wave vectors $0<q<k$ in the integral of equation (4).

In the second case $q>k$, the electromagnetic wave is no longer sensitive to the spatial variation of the fluctuations. The sharp but rough boundary has the same space averaged density profile as a superposition of horizontal layers of uniform densities $\rho(z)$ given by equation (3) in which $L=\left\langle\zeta_{2}^{2}\right\rangle^{1 / 2}$ represents the contribution of the wave vectors $k<q<q_{\max }$ in equation (4) : $\left\langle\zeta^{2}\right\rangle=\left\langle\zeta_{1}^{2}\right\rangle+\left\langle\zeta_{2}^{2}\right\rangle$.

The reflectivity of the equivalent diffuse interface is given by [5] :

$$
R=R_{\mathrm{F}}\left[\frac{1}{\left(\rho_{1}-\rho_{2}\right)} \int_{-\infty}^{+\infty} \frac{\mathrm{d} \rho}{\mathrm{d} z} \mathrm{e}^{2 i k z} \mathrm{~d} z\right]^{2} .
$$

The reflectivity loss due to these last fluctuations is finally found to be :

$$
R_{2}=R_{\mathrm{F}} \mathrm{e}^{-4 k^{2}\left\langle\zeta_{2}^{2}\right\rangle}
$$

This is in agreement with the work of P. Bousquet [10] relative to diffraction gratings of periodicities smaller than $\lambda$.

Generalizing further the result of Beckmann and Spizzichino one can replace in equation (5), the reflectivity $R_{\mathrm{F}}$ by the reflectivity $R_{2}$ corrected for the contributions of the fluctuations of large wavelengths (Eq. (7)). Finally the complete expression of the reflectivity is given by $\left({ }^{4}\right)$ :

$$
R=R_{\mathrm{F}} \mathrm{e}^{-4 k^{2}\left\langle\zeta^{2}\right\rangle} .
$$

In the BLS theory, the whole fluctuations are replaced by their space averaged profile : equation (3) with $L=\left\langle\zeta^{2}\right\rangle^{1 / 2}$. By combining equations (3) and (6), these authors already obtained the reflectivity of equation (8). However this procedure is not rigourous : the multilayer model is not appropriate to describe fluctuations of wavelengths larger than $\lambda$ since it does not predict any scattered light. Fortuitously it leads to the same loss of reflectivity as the exact treatment presented above. But conversely the transmission coefficient $T=1-R$ is erroneous : the exact solution is $T=1-R-Q, Q$ being the total relative scattered intensity.

In the following we will see that the measured reflectivity can be smaller than that predicted by equation (8). This discrepancy with BLS theory can be attributed to supplementary contributions to interfacial thickness arising from a real density profile as first predicted by Van der Waals. They will superimpose at the interface to form a thicker transition region. The reflectivity of the interface which is now both rough and diffuse becomes extremely difficult to calculate. However

$\left({ }^{3}\right)$ Equation (5) no longer holds when surface roughness is too large. But it is expected to be valid down to $\gamma \sim 10^{-3} \mathrm{dyn}$. $/ \mathrm{cm}$, where the average slope of the surface is of the order of 0.1 [9].

$\left({ }^{4}\right)$ Result found independently by P. G. de Gennes (lectures at the Collège de France, 1981). 
there is still a very simple case when the real density profile is described by the error function profile : equation (3) with $L=L_{\mathrm{p}}, L_{\mathrm{p}}$ being now an « intrinsic » interfacial thickness. The reflectivity of the interface is then, by generalizing equation (8) :

$$
R=R_{\mathrm{F}} \mathrm{e}^{-4 k^{2} L^{2}}
$$

with

$$
L^{2}=\left\langle\zeta^{2}\right\rangle+L_{\mathrm{p}}^{2}
$$

It has been noted by W. W. Webb and collaborators that the error function profile : equation (3) for $L=L_{\mathrm{p}}$, is very close to the Fisk and Widom profile : equation (2) for $L=L_{\mathrm{p}} \sqrt{2 \pi}$. Therefore one can expect that even for a Fisk and Widom profile equations (9) and (10) are good approximations. Moreover the scaling predictions give in that case

$$
L_{\mathrm{p}} \sim \frac{2 \sqrt{6}}{\sqrt{2 \pi}} \xi^{\prime} \sim 2 \xi^{\prime} .
$$

3. Reinterpretation of previous results. - W. W. Webb and collaborators included part of the scattered light in their specular reflectivity measurements. The data analysis can be done simply by applying equation (9) but replacing equation (10) by

$$
L^{2}=\left\langle\zeta^{\prime 2}\right\rangle+L_{\mathrm{p}}^{2}
$$

where $\left\langle\zeta^{\prime 2}\right\rangle$ represents the contributions of the wave vectors $q_{\min }<q<q_{\max }$ in the integral of equation (3); $q_{\min }=k \sin \theta, \theta$ being the collection angle. In this way, we subtract the contributions of the wave vectors $0<q<q_{\min }$ that scatters light into the aperture used to collect the specular reflection.

We have reported in table I part of the data of W. W. Webb and collaborators and we compare them with our predictions. It appears from the table that the BLS model cannot account for the total interfacial thickness value. However, the contribution of the thermal fluctuations cannot

Table I. - Comparison between measured L values (Refs. [5, 7] and Eq. (9)), intrinsic thickness values $L_{\mathbf{p}}$ (according to the theory presented in this paper :

$$
L_{\mathrm{p}}=\left\{L^{2}-\frac{k_{\mathrm{B}} T}{4 \pi \gamma} \ln \left[\frac{\left(l_{\mathrm{c}} / L\right)^{2}+1}{l_{\mathrm{c}}^{2} q^{2} \theta^{2}+1}\right]\right\}^{1 / 2},
$$

with $L, \gamma, \Delta \rho$ data of references $[5,7,11,12,13]$ and $\theta=10^{-2}$ rad. [16]) and correlation length values above $T_{\mathrm{c}}$ (from Refs. $\left.[5,11]\right)$. Theory predicts $\xi_{0}^{\prime} \sim 1.5 \xi_{0}[5] ; \varepsilon=T_{\mathrm{c}}-T / T_{\mathrm{c}}$.

\begin{tabular}{|c|c|c|c|c|}
\hline System & $|\varepsilon|$ & $10^{-2}$ & $10^{-3}$ & $10^{-4}$ \\
\hline \multirow{3}{*}{$\mathrm{SF}_{6}$} & $L$ & 72 & 301 & 1250 \\
& $L_{\mathbf{p}}$ & 3.5 & 166 & 786 \\
& $\xi$ & 25 & 101 & 413 \\
\hline \multirow{3}{*}{ Cyclohexane-methanol } & $L$ & 87 & 403 & 1860 \\
& $L_{\mathbf{p}}$ & 77 & 374 & 1780 \\
& $\xi$ & 18 & 85 & 392 \\
\hline
\end{tabular}


be neglected specially for the case of $\mathrm{SF}_{6}$ samples where it is dominant. In the case of cyclohexanemethanol mixtures their contribution are comparable with the claimed uncertainty.

4. Multicomponent mixtures. - We have studied five component mixtures : water + sodium chloride (46.8 wt. \%), toluene $(47.2 \%)$, pentanol $1(4 \%)$ and sodium dodecyl sulfate $(2 \%)$. According to the water salinity $S$ these mixtures separate into two or three phases : $3.5<S<5.4 \mathrm{wt}$. \%, an oil in water microemulsion in equilibrium with an organic phase, $5.4<S<7.4 \mathrm{wt}$. $\%$, a microemulsion in equilibrium with an aqueous and an organic phase and $7.4<S<10 \mathrm{wt}$. \%, a water in oil microemulsion in equilibrium with an aqueous phase [14]. The phase boundaries $S_{1}=5.4$ and $S_{2}=7.4 \mathrm{wt}$. \% are close to critical consolute points.

We have done reflectivity measurements on the planar interface between two phases, one or both being a microemulsion. The samples are temperature controlled at $20 \pm 0.1{ }^{\circ} \mathrm{C}$. The reproducibility of reflectivity measurements is within $30 \%$. The corresponding uncertainty on $L$ becomes large at small $L: 5 \%$ for $L=500 \AA, 30 \%$ for $L=200 \AA$; interfacial thicknesses below $100 \AA$ cannot be measured. In these measurements the solid angle of detection is a cone of smaller aperture $\left(10^{-3} \mathrm{rad}\right.$.) than in previous measurements : the scattered light included in the reflectivity measurements is negligibly small.

The results are reported in table II together with values of $\left\langle\zeta^{2}\right\rangle$ calculated from interfacial tensions and densities measured on the same samples [14]. It must be noted that the choice of $q_{\max }$ is not crucial : if $q_{\max }$ changes by a factor $10, L$ changes by less than $10 \%$. The results do not appear to depend on microemulsion particle size. This is analogous to simpler micellar solutions where the surfactant molecules form a monolayer at the surface of the solution. When the critical points are approached in our liquid mixtures, we have already verified that the particle size does not increase, whereas interfacial tension, interfacial thickness, bulk properties like osmotic compressibility diffusion coefficient, ... behave as predicted by the theory of critical phenomena [14].

Table II. - Comparison between our measurements and BLS theory.

\begin{tabular}{|l|c|c|c|c|}
\hline \multirow{2}{*}{$S$} & \multicolumn{2}{|c|}{ Microemulsion-organic phase } & \multicolumn{2}{c|}{ Microemulsion-aqueous phase } \\
\cline { 2 - 5 } & $L$ & $\left\langle\zeta^{2}\right\rangle^{1 / 2}$ & $L$ & $\left\langle\zeta^{2}\right\rangle^{1 / 2}$ \\
\hline 5.3 & $153( \pm 75)$ & 222 & & \\
5.6 & $207( \pm 60)$ & 260 & & \\
5.7 & & & $602( \pm 20)$ & 601 \\
5.9 & $267( \pm 45)$ & 303 & $467( \pm 25)$ & 464 \\
6 & $392( \pm 30)$ & 396 & $299( \pm 40)$ & 427 \\
6.5 & $540( \pm 20)$ & 582 & $242( \pm 50)$ & 255 \\
7 & & & $204( \pm 60)$ & 227 \\
7.2 & & & $161( \pm 75)$ & 213 \\
7.5 & & &
\end{tabular}

It appears from table II that the data are in good agreement with the BLS model, i.e. $L \sim\left\langle\zeta^{2}\right\rangle^{1 / 2}$. This result is not in contradiction with Fisk and Widom predictions $L_{\mathrm{p}} \sim 2.5 \xi^{\prime}$. In these systems the correlation length $\xi^{\prime}$, as deduced from bulk light scattering measurements [14], is very small : $L / L_{\mathrm{p}} \sim 6$, leading to small contributions of $L_{\mathrm{p}}$ in equation (10).

5. Conclusion. - The interpretation of optical reflectivity measurements of thick liquid interfaces is more complex than previously reported. A large contribution to the reflectivity loss arises 
from capillary waves thermally excited at the interface. This explains why the interfacial profile is well described by the error function.

It can be noted that the corresponding contribution to the thickness $\left\langle\zeta^{2}\right\rangle^{1 / 2}$ does not follow a scaling law : although scaling theories predict $\gamma \sim \varepsilon^{2 v}$, the logarithm in equation (4) involves two different lengths, $L$ that becomes infinite at the critical point, and $l_{\mathrm{c}}=\sqrt{\gamma / \Delta \rho g}$ that goes to zero $\left(\Delta \rho \sim \varepsilon^{\beta}, \beta=0.33\right)$. Let us note however that in practice the variation of the logarithm is small, and this anomaly is impossible to detect experimentally over the covered range of $\varepsilon$. The resulting effective thickness $L$ (Eq. (10)), if the small variations of the logarithm are neglected, follows therefore a scaling law $L=L_{0} \varepsilon^{v^{\prime}}$. This was verified in all the reported experiments [5, 7] (Fig. 2).

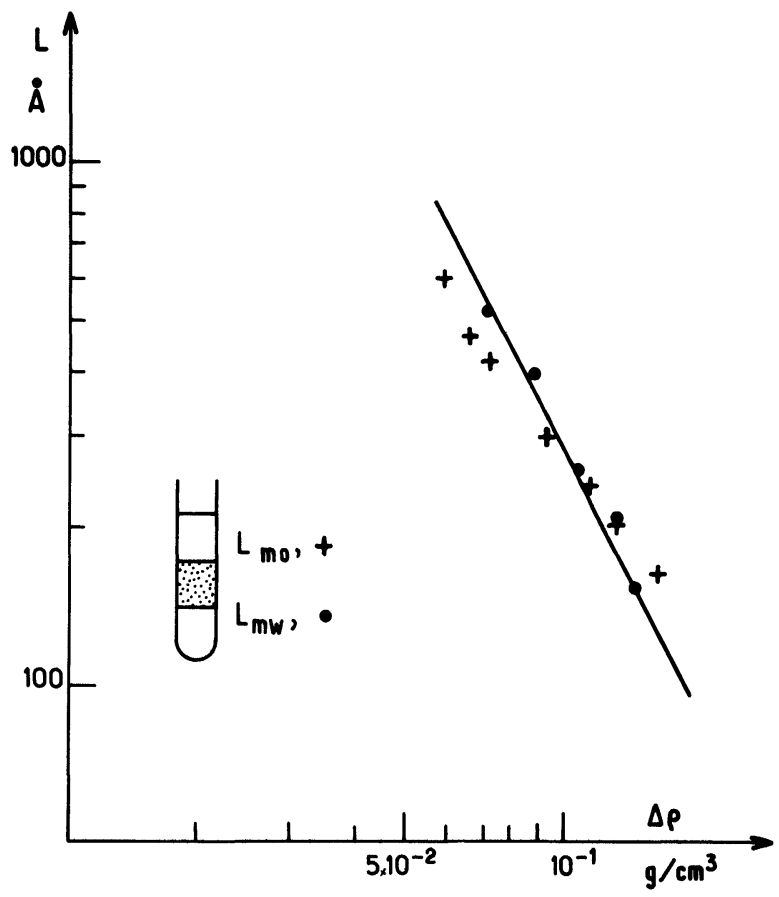

Fig. 2. - Log-Log plot of measured interfacial thickness versus $\Delta \rho$. The line is theoretical and its slope is 2 (from $L \sim \varepsilon^{v^{\prime}}$ and $\Delta \rho \sim \varepsilon^{\beta}$, one has : $L \sim \Delta \rho^{v^{\prime} / \beta}=\Delta \rho^{2}$ ).

The contribution to the reflectivity loss arising from the intrinsic density profile is found to be in agreement with Fisk and Widom's prediction except perhaps for $\mathrm{SF}_{6}$ where the experimental values of $L_{\mathrm{p}}$ are too small. Let us note however that after subtraction of the thermal fluctuations contribution, the experimental accuracy becomes very poor to investigate the remaining contributions to the density profile. Further work is certainly needed to understand the nature of this contribution.

Let us finally mention that a similar approach was carried out in a much smaller interfacial thickness range, for the liquid vapour interface of other pure liquids (argon, carbon tetrachloride) far from their critical points, as studied by ellipsometry [15]. In these cases also, the contribution of thermal fluctuations seems dominant.

Acknowledgments. - We are greatly indebted to P.G. de Gennes for extremely useful discussions and to W. W. Webb for very helpful comments during the elaboration of the manuscript. 


\section{References}

[1] VAN der WaAls, J., Z. Phys. Chem. 13 (1894) 657.

[2] Cahn, J. W., Hilliard, J. E., J. Chem. Phys. 28 (1958) 258.

[3] Fisk, S., Widom, B., J. Chem. Phys. 50 (1969) 3219.

[4] Buff, F. P., Lovett, R. A., Stillinger Jr., F. H., Phys. Rev. Lett. 15 (1963) 621.

[5] Wu, E. S., Webb, W. W., Phys. Rev. A 8 (1973) 2065.

[6] Gilmer, G. M., Gilmore, W., Huang, J., WebB, W. W., Phys. Rev. Lett. 14 (1965) 491.

[7] Huang, J. S., WebB, W. W., J. Chem. Phys. 50 (1969) 3677.

[8] Beckmann, P., Sizzichino, A., The scattering of electromagnetic waves from rough surfaces (Pergamon) 1963.

[9] Meunier, J., C.R. Hebd. Séan. Acad. Sci. 292 II (1981) 1469.

[10] BousQuet, P., Rev. Opt. 41 (1962) 277.

[11] Warren, C., WebB, W. W., J. Chem. Phys. 50 (1969) 3694.

[12] Wu, E. S., Webi, W. W., Phys. Rev. A 8 (1973) 2077.

[13] Balzarini, D., Ohrn, K., Phys. Rev. Lett. 29 (1972) 840.

[14] Cazabat, A. M., Langevin, D., Meunier, J., Pouchelon, A., Adv. Colloid Interface Sci. and J. Physique Lett. 43 (1982) L-89.

[15] Beaglehole, D., Physica 100B (1980) 163.

[16] WeBB, W. W., private communication. 